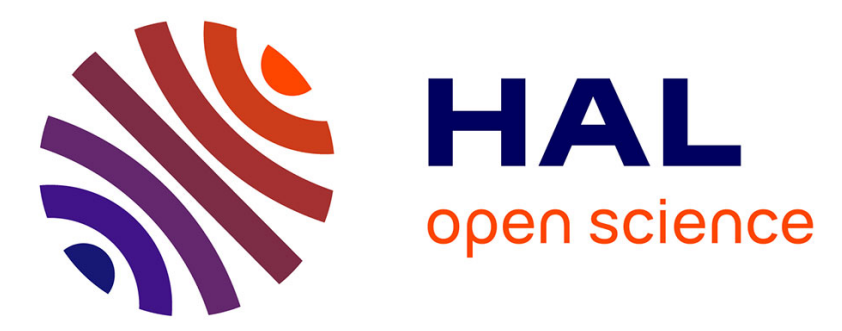

\title{
Replica derivation of Sompolinsky free energy functional for mean field spin glasses
}

Cirano de Dominicis, M. Gabay, Henri Orland

\section{To cite this version:}

Cirano de Dominicis, M. Gabay, Henri Orland. Replica derivation of Sompolinsky free energy functional for mean field spin glasses. Journal de Physique Lettres, 1981, 42 (23), pp.523-526. 10.1051/jphyslet:019810042023052300 . jpa-00231991

\section{HAL Id: jpa-00231991 https://hal.science/jpa-00231991}

Submitted on 1 Jan 1981

HAL is a multi-disciplinary open access archive for the deposit and dissemination of scientific research documents, whether they are published or not. The documents may come from teaching and research institutions in France or abroad, or from public or private research centers.
L'archive ouverte pluridisciplinaire HAL, est destinée au dépôt et à la diffusion de documents scientifiques de niveau recherche, publiés ou non, émanant des établissements d'enseignement et de recherche français ou étrangers, des laboratoires publics ou privés. 


\title{
Replica derivation of Sompolinsky free energy functional for mean field spin glasses
}

\author{
C. de Dominicis, M. Gabay (*) and H. Orland \\ Service de Physique Théorique, CEN Saclay, 91191 Gif sur Yvette Cedex, France
}

(Reçu le 28 août 1981, accepté le 12 octobre 1981)

\begin{abstract}
Résumé. - Nous établissons une forme de la fonctionnelle d'énergie libre pour les verres de spin dans la limite du champ moyen que Sompolinsky a obtenue récemment via une approche dynamique. Nous utilisons ici la méthode des répliques avec une division en blocs analogue à celle employée pour établir la solution de Sommers et avec une procédure d'iteration proche de celle de Parisi mais portant sur les blocs diagonaux et hors diagonaux.

Abstract. - We derive a form of the free energy functional for mean field spin glasses that has been recently obtained by Sompolinsky via a dynamic approach. Here we use replicas with a block division along the lines used to derive Sommers solution and with an iterative procedure close to that of Parisi applied to both off diagonal and diagonal blocks.
\end{abstract}

1. Introduction. - The infinite ranged spin glass model of Sherrington and Kirkpatrick [1] (SK) that describes a mean field approximation to real spin glasses, has given rise to abundant work but has resisted so far, a full understanding. Even though the early description of the gelation transition was associated with anomalous behaviour of the large time limit of spin correlation functions [2], most of the work has been done with a static approach. In particular, but, by large, not exclusively, attention has been focused on trying to solve this model by using a replica trick $[2,3]$ that allows to directly take "quenched" averages at the price of having, in the end, to take the unphysical limit of the number $n$ of replicas going to zero.

The so called SK solution describes the transition with a single, replica independent, Edwards Anderson (EA) order parameter

$$
q_{0}=\overline{\left\langle\sigma_{i}^{\alpha} \sigma_{i}^{\beta}\right\rangle}, \quad \alpha \neq \beta .
$$

Here the thermodynamic average $\langle>$ is taken with a weight $\exp -\beta H$

$$
H=-\sum_{(i, j)} J_{i j} \sigma_{i} \sigma_{j}
$$

the sum being over all pairs of sites $(i, j), \sigma_{i}$ an Ising spin. The bar stands for average over the gaussian distribution of bonds

$$
P\left(J_{j l}\right) \simeq \exp -N J_{j l}^{2} / 2 J^{2}
$$

and $\alpha, \beta$ are replica indices $(\alpha=1,2, \ldots, n)$.

The SK solution unfortunately leads to unphysical results [1] and is indeed unstable [4] with respect to fluctuations. A distinct solution has been exhibited by Sommers [5] that involves, besides the EA order parameter $q_{0}$, an anomaly $a_{0}$ to the linear response function. In terms of the replica approach, this solution is identified $[6,7]$ with a limiting case of an extension to the Blandin et al. [8] symmetry breaking scheme. One introduces an $n \times n$ order parameter matrix

$$
q_{\alpha \beta}=\left(\begin{array}{c|c|c}
q_{0} & r_{0} & r_{0} \\
\hline r_{0} & q_{0} & r_{0} \\
\hline r_{0} & r_{0} & q_{0}
\end{array}\right) .
$$

Here there are $\left(n / p_{0}\right)^{2}$ constant blocks, each one of size $p_{0} \times p_{0}$, with value $q_{0}$ for a diagonal, $r_{0}$ for an off diagonal block. The limit $p_{0} \rightarrow \infty$ is taken after $n \rightarrow 0$. In that limit 
is the EA order parameter, and the anomaly, for consistency with reference [12], is

$$
-\Delta_{0}^{\prime} \underset{p_{0} \rightarrow \infty}{=} p_{0}\left(q_{0}-r_{0}\right), \quad \Delta_{0}^{\prime}<0 .
$$

Sommers solution, even though it does not carry distasteful features of the SK solution, remains unstable [6].

In a bold generalization, and guided by requirements of positive definiteness on the free energy, Parisi [9] has introduced a symmetry breaking scheme that leads to remarkable physical properties [ $9 b]$ and verifies some stability criteria [10]. Parisi defines a self similar iterative procedure to build $q_{\alpha \beta}$ :

The starting (zero) step being the constant, $n \times n$, $q_{0}$ matrix, step one is obtained by the transform $T$

$$
\left(q_{0}\right) \overrightarrow{\vec{r}}\left(\begin{array}{c|c|c}
q_{1} & q_{0} & q_{0} \\
\hline q_{0} & q_{1} & q_{0} \\
\hline q_{0} & q_{0} & q_{1}
\end{array}\right)
$$

characterized by $\left(t_{i}\right)$ a division into $\left(n / m_{1}\right)^{2}$ blocks, each one $m_{1} \times m_{1}$ in size, $\left(t_{i i}\right)$ a shift $\left(q_{0} \rightarrow q_{1}\right)$ in the value of the diagonal blocks only. The transform is iterated $\left(t_{i}\right)$ each $q_{1}$ block is divided into $\left(m_{1} / m_{2}\right)^{2}$ blocks of size $m_{2} \times m_{2}$ and $\left(t_{i i}\right)$ the new diagonal blocks are shifted $\left(q_{1} \rightarrow q_{2}\right)$. And so on, with the restriction

$$
1 \leqslant m_{K} \leqslant \cdots \leqslant m_{2} \leqslant m_{1} \leqslant n .
$$

When $n \rightarrow 0$, the continuous limit is obtained by

and

$$
m_{j}=j /(K+1), \quad K \rightarrow \infty
$$

$$
q_{j}=q(x), \quad m_{j}<x<m_{j+1} .
$$

Questions left open include (i) are Parisi iteration and solution unique, (ii) what is the physical meaning of the parameter $x$ [11].

In a far reaching recent paper, Sompolinsky [12] has reattacked the problem from a dynamical point of view. He has come out with a description that involves a double continuum of order parameters $q(x)$ and $\Delta^{\prime}(x)$, the $x$ index labelling now the physical continuum of infinite relaxation times that characterize the system. He ends up with an explicit functional of $q(x)$ and $\Delta^{\prime}(x)$ that contains the main features of Parisi solution.

In this note we show that one recovers Sompolinsky functional by taking Sommers matrix (4) as a zero step, and applying, both on the diagonal $\left(q_{0}\right)$ and off diagonal $\left(r_{0}\right)$ blocks a self similar iterative procedure described below.

2. Step zero : Sommers free energy functional $[6,7]$. - The free energy functional for the SK model writes

$$
-\beta f=\frac{\beta^{2} J^{2}}{4}+\left.\frac{\partial}{\partial n}\right|_{n=0} \operatorname{Max}\left[-\frac{\beta^{2} J^{2}}{4} \sum_{\alpha \neq \beta} q_{\alpha \beta}^{2}+\ln \operatorname{Tr}_{\sigma} \exp \frac{\beta^{2} J^{2}}{2} \sum_{\alpha \neq \beta} q_{\alpha \beta} \sigma_{\alpha} \sigma_{\beta}\right] .
$$

It is readily evaluated when $q_{\alpha \beta}$ has the structure (4) via

$$
\begin{aligned}
\sum_{\alpha \neq \beta} q_{\alpha \beta}^{2} & =r_{0}^{2} n(n-1)+\left(q_{0}^{2}-r_{0}^{2}\right) n\left(p_{0}-1\right) \\
\sum_{\alpha \neq \beta} q_{\alpha \beta} \sigma_{\alpha} \sigma_{\beta} & =r_{0}\left(\sum_{j_{0}, \alpha} \sigma_{j_{0}, \alpha}\right)^{2}+\left(q_{0}-r_{0}\right) \sum_{j_{0}=1}^{n / p_{0}}\left(\sum_{\alpha=1}^{p_{0}} \sigma_{j_{0}, \alpha}\right)^{2}-n q_{0} .
\end{aligned}
$$

Here each spin is indexed by the block number $j_{0}=1,2, \ldots, n / p_{0}$ and inside each block by $\alpha=1,2, \ldots, p_{0}$. Using $z_{0}$ and $y_{j_{0}}$ to linearize (13), after taking traces over spins, we obtain for the log term of (11)

$$
\ln \int \frac{\mathrm{d} z_{0}}{(2 \pi)^{1 / 2}} \mathrm{e}^{-z_{0}^{2} / 2} \prod_{j_{0}=1}^{n / p_{0}}\left(\int \frac{\mathrm{d} y_{j_{0}}}{(2 \pi)^{1 / 2}} \exp \left\{-\frac{y_{j_{0}}^{2}}{2}+p_{0} \ln 2 \cosh \left\{\beta J z_{0} r_{0}^{1 / 2}+\beta J y_{j_{0}}\left(q_{0}-r_{0}\right)^{1 / 2}\right\}\right\}\right) .
$$

If we use the anomaly $-\Delta_{0}^{\prime}$ as given by (6), we can, in the limit $p_{0} \rightarrow \infty$ (taken after $\left.n \rightarrow 0[6,7]\right)$ write the $y$ integrals $\left(y_{j_{0}} \rightarrow p_{0}^{1 / 2} y_{j_{0}}\right)$ as saddle point contributions with

$$
y_{j_{0}}^{\mathrm{c}} \equiv \beta J m_{0}\left(z_{0}\right)\left(-\Delta_{0}^{\prime}\right)^{1 / 2} .
$$

The free energy functional follows

$$
\begin{aligned}
-\beta f=\frac{\beta^{2} J^{2}}{4}\left[\left(q_{0}-1\right)^{2}+2 q_{0} \Delta_{0}^{\prime}\right]+ & \int \frac{\mathrm{d} z_{0}}{\left(2 \pi J^{2} q_{0}\right)^{1 / 2}} \mathrm{e}^{-z^{2} / 2 J^{2} q_{0}} \times \\
& \times\left\{+\frac{\beta^{2} J^{2}}{2} m_{0}^{2} \Delta_{0}^{\prime}+\ln 2 \cosh \left\{\beta z_{0}-\beta^{2} J^{2} m_{0} \Delta_{0}^{\prime}\right\}\right\} .
\end{aligned}
$$


Here we have used (5). The order parameters $q_{0}, \Delta_{0}^{\prime}$ and the local magnetization $m_{0}\left(z_{0}\right)$, are obtained by stationarity on (15),

$$
\begin{aligned}
m_{0}\left(z_{0}\right) & =\tanh \left[\beta z_{0}-\beta^{2} J^{2} m_{0}\left(z_{0}\right) \Delta_{0}^{\prime}\right] \\
q_{0} & =\int \frac{\mathrm{d} z_{0}}{\left(2 \pi J^{2} q_{0}\right)^{1 / 2}} \mathrm{e}^{-z_{0}^{2} / 2 J^{2} q_{0}} m_{0}^{2}\left(z_{0}\right) \\
\beta\left(1-q_{0}-\Delta_{0}^{\prime}\right) & =\int \frac{\mathrm{d} z_{0}}{\left(2 \pi J^{2} q_{0}\right)^{1 / 2}} \mathrm{e}^{-z_{0}^{2} / 2 J^{2} q_{0}} \frac{\partial}{\partial z_{0}} m_{0}\left(z_{0}\right) .
\end{aligned}
$$

Introducing an external magnetic field $h$, adds a $\beta h$ term inside the $\ln$ cosh, thus identifying $-\Delta_{0}^{\prime}$ as the anomaly.

Note that the above results are also obtained for the reverse order of limits i.e. $p_{0} \rightarrow \infty$ and then, $n=0$.

3. Iteration procedure : step one. - We apply iteration (7) both on off diagonal blocks $r_{0}$ (with shift $r_{1}$ on the diagonal subblocks) and on diagonal blocks $q_{0}$ (shift $q_{1}$ ). Each spin is now indexed by block number $j_{0}=1,2, \ldots, n / p_{0}$ and subblock number $j_{1}=1,2, \ldots, p_{0} / p_{1}$ (for a given $j_{0}$ ), and inside each subblock by $\alpha=1,2, \ldots, p_{1}$. For step zero we had $p_{0} \rightarrow \infty$. Here $p_{0} \gg p_{1}$ and both go to infinity in succession. This procedure differs from Parisi's in two respects (i) it applies to both diagonal and off diagonal blocks, (ii) the division procedure $\left(p_{i} \rightarrow \infty\right)$ leaves no variational parameters as is the case for Parisi $m_{i}$ 's.

With the above instructions equation (13) becomes

$$
\begin{aligned}
\sum_{\alpha \neq \beta} q_{\alpha \beta} \sigma_{\alpha} \sigma_{\beta}=r_{0}\left(\sum_{j j_{1} \alpha} \sigma_{j_{0 j} j_{1} \alpha}\right)^{2}+\left(q_{0}-r_{0}\right) \sum_{j_{0}}\left(\sum_{j_{1} \alpha} \sigma_{j_{0} j_{1} \alpha}\right)^{2}-n q_{1}+ \\
+\left(r_{1}-r_{0}\right) \sum_{j_{1}}\left(\sum_{j_{0} \alpha} \sigma_{j_{0} j_{1} \alpha}\right)^{2}+\left[\left(q_{1}-q_{0}\right)-\left(r_{1}-r_{0}\right)\right] \sum_{j_{0} j_{1}}\left(\sum_{\alpha} \sigma_{j_{0} j_{1 \alpha} \alpha}\right)^{2} .
\end{aligned}
$$

We introduce variables $z_{0}$ and $z_{j_{1}}$ to unfold terms in $r_{0}$ and $\left(r_{1}-r_{0}\right)$, variables $y_{j_{0}}$ and $y_{j_{0} j_{1}}$ for terms in $\left(q_{0}-r_{0}\right)$ and $\left[\left(q_{1}-q_{0}\right)-\left(r_{1}-r_{0}\right)\right]$. Using (6) and

$$
-\Delta_{1}^{\prime}=p_{1}\left[\left(q_{1}-q_{0}\right)-\left(r_{1}-r_{0}\right)\right]
$$

we get for the log term of (11)

$$
\begin{aligned}
\ln \int \frac{\mathrm{d} z_{0}}{(2 \pi)^{1 / 2}} \mathrm{e}^{-z z_{0}^{2} / 2} \int \prod_{j_{0}}\left(\frac{\mathrm{d} y_{j_{0}}}{(2 \pi)^{1 / 2}} \mathrm{e}^{-p_{0} y_{j_{0}}^{2} / 2}\right) \prod_{j_{1}=1}^{p_{0} / p_{1}}\left\{\int \frac{\mathrm{d} z_{j_{1}}}{(2 \pi)^{1 / 2}} \mathrm{e}^{-z_{j_{1}}^{2} / 2} \times\right. \\
\quad \times \int_{j_{0}=1}^{n / p_{0}}\left(\frac { \mathrm { d } y _ { j _ { 1 } j _ { 0 } } } { ( 2 \pi ) ^ { 1 / 2 } } \operatorname { e x p } \left\{-p_{1} y_{j_{0} j_{1}}^{2} / 2+p_{1} \ln 2 \cosh \left[\beta J z_{0} r_{0}^{1 / 2}+\beta J z_{j_{1}}\left(r_{1}-r_{0}\right)^{1 / 2}\right.\right.\right. \\
\left.\left.\left.\left.+\beta J\left[y_{j_{0}}\left(-\Delta_{0}^{\prime}\right)^{1 / 2}+y_{j_{0} j_{1}}\left(-\Delta_{1}^{\prime}\right)^{1 / 2}\right]\right]\right\}\right)\right\}
\end{aligned}
$$

Here we see that it is essential now that the limits be taken in the order (i) $p_{0} \rightarrow \infty$, (ii) $p_{1} \rightarrow \infty$, (iii) only then $n \rightarrow 0$. In this order, the saddle point values (14) and $\beta J m_{1}\left(z_{0}, z_{1}\right)\left(-\Delta_{1}^{\prime}\right)^{1 / 2}$ for $y_{j_{j} j_{1}}^{c}$ are given by

$$
m_{1}=\tanh \left\{\beta J\left[z_{0} q_{0}^{1 / 2}+z_{1}\left(q_{1}-q_{0}\right)^{1 / 2}\right]-\beta^{2} J^{2}\left[m_{0} \Delta_{0}^{\prime}+m_{1} \Delta_{1}^{\prime}\right]\right\}
$$

in which we have used (5) and $r_{1}=q_{1}$, together with

$$
m_{0}=\int \frac{\mathrm{d} z_{1}}{(2 \pi)^{1 / 2}} \mathrm{e}^{-z_{1}^{2} / 2} m_{1} .
$$

In deriving (23) we have used the fact that the weight

$$
\exp p_{1} \sum_{j_{0}}\left[+m_{j_{0} 1}^{2} \Delta_{1}^{\prime} / 2+\ln 2 \cosh \left[\beta J\left[z_{0} q_{0}^{1 / 2}+z_{1}\left(q_{1}-q_{0}\right)^{1 / 2}\right]-\beta^{2} J^{2}\left[m_{j_{0}} \Delta_{0}^{\prime}+m_{j_{0} 1} \Delta_{1}^{\prime}\right]\right]\right]
$$

disappears with the factor $\sum_{j_{0}} \equiv n / p_{0}$ when taken at the saddle point value with all $m_{j_{0} 1} \equiv m_{1}$. 
The free energy functional replacing (15) is now

$$
\begin{aligned}
-\beta f=\frac{\beta^{2} J^{2}}{4}\left[\left(q_{1}-1\right)^{2}+\right. & \left.2 \sum_{j=0}^{1} q_{j} \Delta_{j}^{\prime}\right]+\int_{j_{0}=0}^{1}\left(\frac{\mathrm{d} z_{j}}{\left[2 \pi J^{2}\left(q_{j}-q_{j-1}\right)\right]^{1 / 2}} \times \exp -z_{j}^{2} / 2 J^{2}\left(q_{j}-q_{j-1}\right)\right) \cdot \\
\cdot & {\left[+\frac{\beta^{2} J^{2}}{2} \sum_{j=0}^{1} m_{j}^{2} \Delta_{j}^{\prime}+\ln 2 \cosh \left\{\beta h+\sum_{j=0}^{1}\left(\beta z_{j}-\beta^{2} J^{2} m_{j} \Delta_{j}^{\prime}\right)\right\}\right] . }
\end{aligned}
$$

We have here written the result in general form with $q_{-1} \equiv 0$. Equations $(23,22)$ derive from stationarity (saddle point) in $m_{0}\left(z_{0}\right), m_{1}\left(z_{0}, z_{1}\right)$, stationarity in $q_{0}, \Delta_{0}^{\prime}$ and $q_{1}-q_{0}, \Delta_{1}^{\prime}$ yield the analog of equations (18) and (17) for $\Delta_{1}^{\prime}$ and $q_{1}$.

4. General form and comments. - The above procedure is trivially repeated (with sequences $q_{1}, q_{2} \ldots$, $\left.q_{k}, r_{1}, r_{2}, \ldots, r_{k}\right)$. The $k$ th iterated result is obtained by replacing index 1 by $k$ in (24) and expressing stationarity in $m_{j}, q_{j}-q_{j-1}, \Delta_{j}^{\prime}$. This is then identical with the discretized form of Sompolinsky [12] free energy functional, definition of the continuous limit being given in that reference.

Several points may be stressed : (i) The order of limits $p_{0} \gg p_{1} \gg \cdots \gg p_{k} \rightarrow \infty$ and then $n \rightarrow 0$ is surprising, the other way around ( $n \rightarrow 0$ first) seeming more "physical». A reformulation of the replica trick in terms of $P \times P, P \rightarrow \infty$ matrices (instead of $n \times n, n \rightarrow 0$ ) that may help to render palatable the above order, is discussed elsewhere [13]. (ii) Contrary to Parisi approach, there is no way here in which results could be vibrational in the parameters $p_{1}$, $p_{2}, \ldots, p_{k}$. (iii) The present derivation may suggest a purely static interpretation of the order parameters that is currently under investigation.

Acknowledgments. - The authors wish to thank B. Duplantier for a critical reading of the manuscript.

\section{References}

[1] Sherrington, D., KirkPatrick, S., Phys. Rev. Lett. 35 (1975) 1792.

KirkPatrick, S., Sherrington, D., Phys. Rev. B 17 (1978) 4384.

[2] Edwards, S. F., Anderson, P. W., J. Phys. F 5 (1975) 965, $F$ (1976) 1927.

[3] Edwards, S. F., Highly Cross Linked Networks (Plenum Press, N.Y.) 1971.

EMERY, V., Phys. Rev. B 11 (1975) 239.

Edwards, S. F., J. Phys. A 13 (1980) L.239.

[4] De Almeida, J. R. L., Thouless, D. J., J. Phys. A 11 (1978) 983.

[5] Sommers, H. J., Z. Phys. B 31 (1978) 301 ; B 33 (1979) 173.

[6] DE Dominicis, C., GAREL, T., J. Physique Lett. 40 (1979) L-575.
[7] Bray, A. J., Moore, M. A., J. Phys. C 13 (1980) 419.

[8] Blandin, A., J. Physique Colloq. 39 (1978) C6-1499.

Blandin, A., Gabay, M., Garel, T., J. Phys. C 13 (1980) 403.

[9] PARISI, G., a) J. Phys. A 13 (1980) L115; A 13 (1980) 1101 ; A 13 (1980) 1887 - b) Philos. Mag. 41 (1980) 677; Phys. Rep. 67 (1980) 25.

[10] Thouless, D. J., De Almeida, J. R. L., Kosterlitz, J. M., J. Phys. C 13 (1980) 3271.

DE AlmeidA, J. R. L., PhD Thesis, Birmingham (1980).

[11] Gabay, M., Garel, T., to appear in J. Phys. A, Comments (Nov. 1981).

[12] Sompolinsky, H., Phys. Rev. Lett. 47 (1981) 935.

[13] ORLand, H., DE Dominicis, C., to be published. 